\title{
Improvement in Absorption of Microwaves by Incorporating Additives in Silica Phenolic Composite
}

\author{
MUHAMMAD Salman ${ }^{1, a^{*}}$, ANWAAR Ellahi ${ }^{2, b}$ and SYED Wilayat Husain ${ }^{1, c}$ \\ ${ }^{1}$ Materials Science and Engineering Department, \\ Institute of Space Technology, Islamabad, Pakistan \\ ${ }^{2}$ Centre of Excellence in Science and Applied Technologies, Islamabad, Pakistan \\ amhmdsalman@live.com, banwaar_ellahi@yahoo.co.uk, 'dr_swh@live.com
}

Keywords: High Density Reactive Material, Microwave Absorption, Composite Modification, Radar Absorbing Materials

\begin{abstract}
A method of adding radar absorbing materials (RAM) directly in silica phenolic composite was tested. Due to direct incorporation of RAM, the resultant composite not only may show microwave absorption at normal conditions but sustain this capability at high heat fluxes. Such RAM added composite becomes more applicable for materials, which are exposed to high temperatures. Two different RAM added silica fiber phenolic composites were developed and their RF-absorptions were compared with pure silica fiber phenolic sample. It was found that depending on the type of RAM their RF-absorption can be enhanced. Strength and ablation properties of the resultant composites were also found to alter with the addition of RAM in the composite.
\end{abstract}

\section{Introduction}

RAM coatings have proved very useful in increasing the microwave absorption of materials. Though these coatings are useful but have certain limitations which hinder their usage especially in extreme environment. Materials used under normal conditions can be coated with RAM paints to enhance and sustain their microwave absorption capability. However, such coatings cannot withstand extreme conditions and erode, therefore are not applicable for materials, which encounter high heat fluxes. The increase in weight of the coated material due to amount of RAM and binder used is another concern. Epoxy or resin is an integral part of a composite and an alternative technique to enhance the microwave absorption capability of a composite can be to incorporate RAM within the composite during its production. Since RAM will be dispersed within the composite and not exposed directly to heat therefore erosion of RAM will be gradual. Thus the microwave absorption of material containing RAM will not be lost instantly when exposed to heat but decrease slowly.

Ablatives encounter harsh environments and protect the internal structure of re-entry vehicles by dissipating heat in the environment rather transferring it to the internal structure. The dissipation of heat is due to charring, which starts when the material is subjected to higher temperature. A thin layer of RAM cannot be practically coated on the surface of this material. However, if RAM becomes an integral part of the ablative then this material can not only act as a heat shield but will also possess microwave absorbing capability even at high temperatures. Ablatives can therefore be modified to enhance their microwave absorption by simply mixing RAM with phenolic resin and using this mixture to produce RAM loaded pre-pegs.

In order to verify the effect of RAM in ablatives, silica fiber phenolic composite samples were produced in which barium ferrite and graphene ferrite were incorporated during their production. Graphene due to its unique properties such as excellent structural flexibility, remarkable electrical conductivity, thermal stability and outstanding mechanical properties has vast applications in many technological fields. Especially in aerospace industry, graphene makes an excellent candidate for electromagnetic shields to absorb incident electromagnetic waves [1]. At high frequencies over $\mathrm{GHz}$ range, graphene is highly desirable to absorb electromagnetic waves due to its low density and high dielectric loss [2]. Ohlan et al. investigated the electromagnetic properties of 
poly $(3,4$ ethylene-di-oxythiophene $) / \mathrm{BaFe}_{12} \mathrm{O}_{19}$ nano composite in the $\mathrm{Ku}$ band frequency region (12.4-18 GHz) and they reported a maximum shielding effectiveness due to absorption of $-22.5 \mathrm{~dB}$ at $15 \mathrm{GHz}$ with minimal reflection loss of $-2 \mathrm{~dB}$ [3]. Xu et al. achieved a maximum reflection loss of $-11.8 \mathrm{~dB}$ at $11.6 \mathrm{GHz}$ by $13.5 \mathrm{wt}$ \% barium ferrite nanoparticles loaded polypyrrole composite with a thickness of $2 \mathrm{~mm}$ [4].

Scanning electron microscopy and X-ray diffraction was done to characterize the RAM powders. Tensile testing was done to evaluate the strength of resultant composites according to ASTM D3039 standard in which specimens are placed in the grips of a universal test machine at a specified grip separation and pulled until failure. The ablation properties and erosion rates of specimens have been carried out in the Plasmatron facility by high-energy air plasma in ambient atmosphere and the mass losses were measured for exposure time of 200 seconds.

The novel effect of incorporating RAM directly in silica fiber Phenolic composite and its effect on absorption was studied in this work. Due to RAM incorporation not only the RF absorption property increased, enhanced ablative properties were also seen. In addition to these properties, mechanical properties like density, strength and back plate temperature are also improved.

\section{Experimental}

Two different types of RAM powders i.e. barium-ferrite $\left(\mathrm{BaFe}_{12} \mathrm{O}_{19}\right)$ and graphene ferrite nanocomposite were developed and used to study the microwave absorption behavior of resulting RAM added silica fiber phenolic composite. Barium ferrite powder was manufactured using sol-gel method [5]. A two-step methodology was used to synthesize graphene ferrite nano-composite. In the first step graphite was oxidized using Hummer's method [6]. The graphite oxide was then used to produce graphene sheets decorated with ferrite nanoparticles [7-8]. In our production technique the reduction process of graphite oxide along with addition of ferrite content (stoichiometric amounts of Co-nitrate and Fe-nitrate) was carried out in a flask (kept at $280^{\circ} \mathrm{C}$ for 6 hours) instead of an autoclave.

Equal amounts of silica fibre and phenolic resin (1:1) were used to synthesize silica fiber phenolic sample. 300 grams of each RAM powder was used with same amounts of silica fiber and phenolic resin to synthesize RAM added composites. In the synthesis process the measured amount of RAM was added and mixed with $1.5 \mathrm{Kg}$ phenolic resin. RAM added resin was mixed with a mixer for half an hour and then $1.5 \mathrm{Kg}$ silica fiber was added and mixed thoroughly.

A total amount of $10 \mathrm{wt} . \%$ of each RAM powder was therefore added in respective composites. This mixture was dried in an oven overnight and once dried these RAM added fibre mixtures were molded at a pressure of $\sim 16 \mathrm{MPa}$ and $165^{\circ} \mathrm{C}$ into composite disks (disks: diameter $19 \mathrm{~mm}$, thickness $\sim 20 \mathrm{~mm}$ ). A pure silica fiber phenolic disc sample was also made for comparison. The compacted samples were then machined into $(6 \times 6$ inch) plates with $10 \mathrm{~mm}$ thickness for RF-measurements. Photos of machined samples are shown in Fig. 1, the variation in colors is due to the type of added RAM powders. Samples for determining tensile strength, ablation and density of composites were acquired from the same composite plates after their microwave absorption measurements were performed.
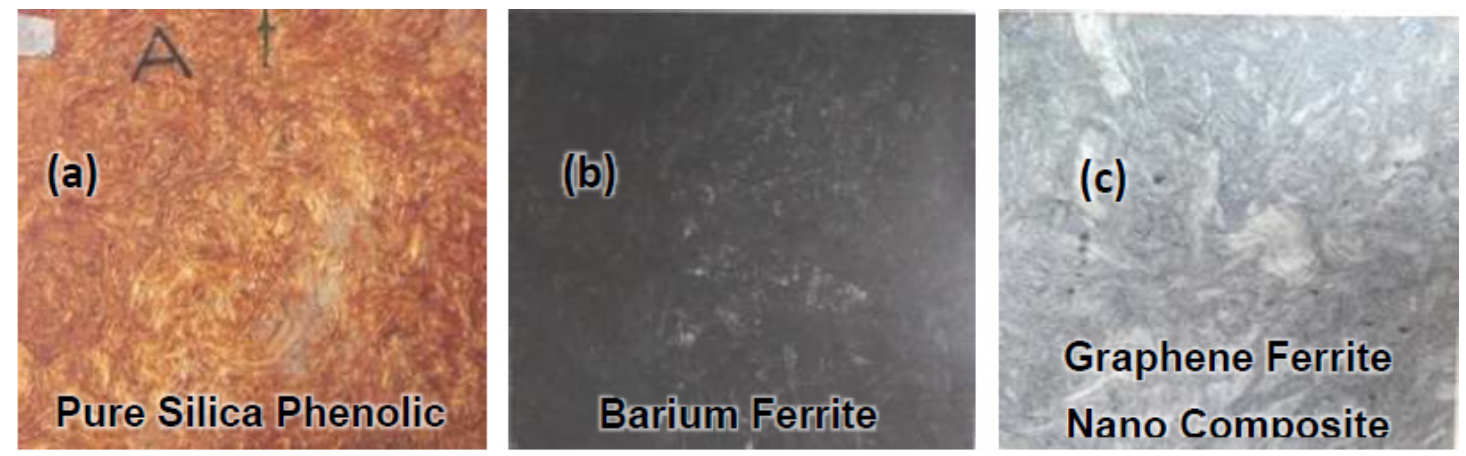

Fig. 1. Photographs of pure (a) silica fibre phenolic and RAM added composites (b) Barium ferrite (c) graphene ferrite 


\section{Results}

SEM images of RAM powders are shown in Fig. $2 \& 3$ revealing their shape and morphology i.e., micron size flakes of hexaferrite (Fig. 2a), exfoliated layers of graphite due to acid treatment and oxidation of graphite (Fig. 2 b-c) and few discrete features ranging in few Nano meters in diameter embedded in graphene sheets (Fig. $3 \mathrm{a}-\mathrm{b}$ ). These features confirm the formation of ferrite nanoparticles within the graphene sheets. Ferrite nanoparticles (av. Size $\sim 20 \mathrm{~nm}$ ) and graphene sheets without any embedded nanoparticles are also visible which show that ferrite content was not totally consumed and resulted in the formation of $\mathrm{CoFe}_{2} \mathrm{O}_{4}$ nanoparticles and graphene. Therefore, this sample is a mixture of graphene ferrite Nano-composite, Co-ferrite nanoparticles and graphene.
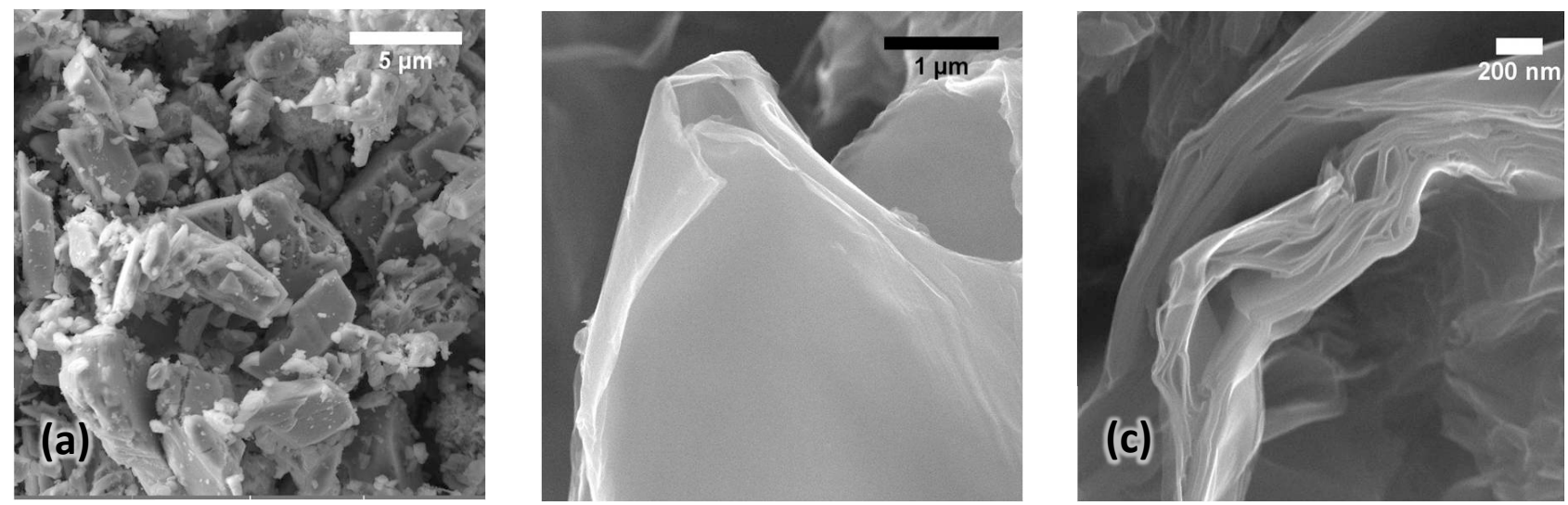

Fig. 2. SEM images of (a) Ba-Ferrite and (b-c) graphite oxide
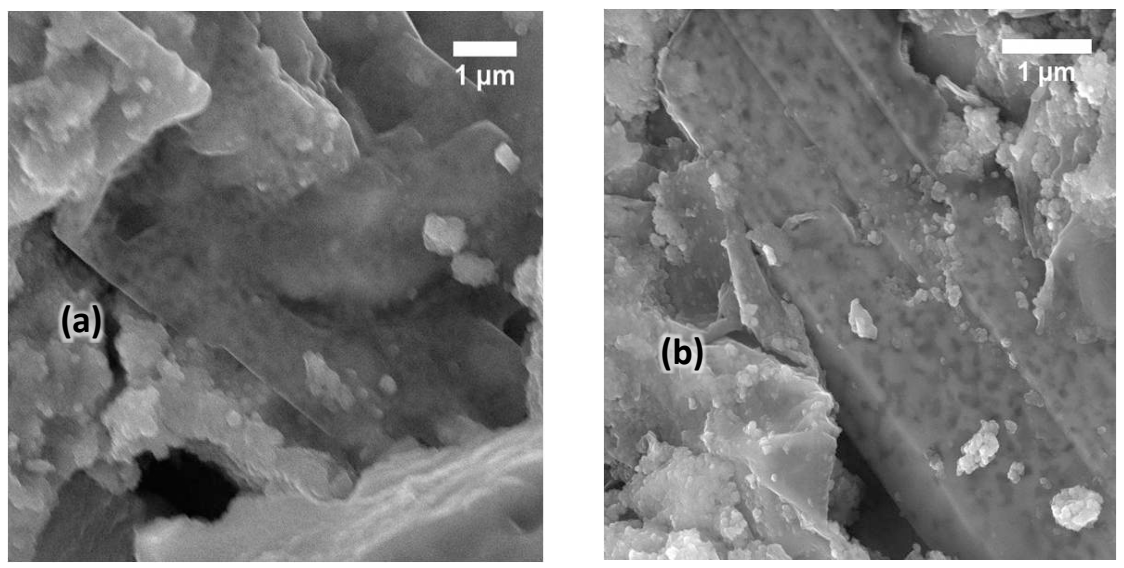

Fig. 3. SEM images of graphene ferrite Nano composite which show ferrite nanoparticles incorporated in graphene as small dark spots. Individual ferrite nanoparticles are also visible

The crystalline phases were identified by X-ray diffraction and the patterns were recorded from $10^{\circ}$ to $80^{\circ}$ with scan rate of $2^{\circ} / \mathrm{min}$. The XRD pattern of Ba-ferrite (Fig. $4 \mathrm{a}$ ) confirms the formation of a single phase M-type hexagonal ferrite since no other phases were detected. XRD pattern of Graphene ferrite Nano-composite (Fig. 4b) show much intense but broader peaks due to Co-ferrite nanoparticles and few weak peaks of graphene. Peaks at $2 \Theta$ values of $30.08^{\circ}, 35.4^{\circ}, 37.08^{\circ}, 43.05^{\circ}$, $53.4^{\circ}, 56.97^{\circ}, 62.58^{\circ}$ and $74.01^{\circ}$ correspond to (220), (311), (222), (400), (422), (511), (440) and (533) planes of cubic Co-ferrite Nano-particles. Only two weaker peaks of graphene at $18.3^{\circ}$ and $26.6^{\circ}$ correspond to (111) and (002) planes while others have been overshadowed due to intense ferrite peaks and therefore are not distinguishable.

Due to machining the original amount of RAM powders mixed in silica fiber phenolic composite disks were reduced. Despite reduction in the amount of RAM our results show that the microwave absorption of RAM added silica fiber phenolic samples is still greater than the pure sample. Microwave absorption result of sample without RAM (black line: Fig. 5) show weak attenuation in $\mathrm{S}$ band, relatively higher attenuation in $\mathrm{X}$ band and strongest attenuation in $\mathrm{K}_{\mathrm{u}}$ band. The Ba-ferrite added composite shows enhanced absorption in all three bands. Whereas modified 
graphene-ferrite added composite gave continuous ( $\geq 70 \%$ ) absorption in $\mathrm{S}, \mathrm{C}, \mathrm{X}$ and $\mathrm{K}_{\mathrm{u}}$ bands and a considerable dip of $-8.9 \mathrm{~dB}$ at $2 \mathrm{GHz}$ (reflection loss $87 \%$ ). This is very promising and shows that this RAM which constitutes mixture of magnetic nanoparticles, embedded ferrite nanoparticles in graphene sheets and graphene can provide broad band absorption. Detailed analyses of absorption, transmission and reflection in the $K_{u}$ band for all the samples are presented in Fig. 6 . These results reconfirm the enhancement of microwave absorption in the $K_{u}$ band with the addition of RAM. In comparison graphene ferrite Nano-composite shows superior absorption capability.
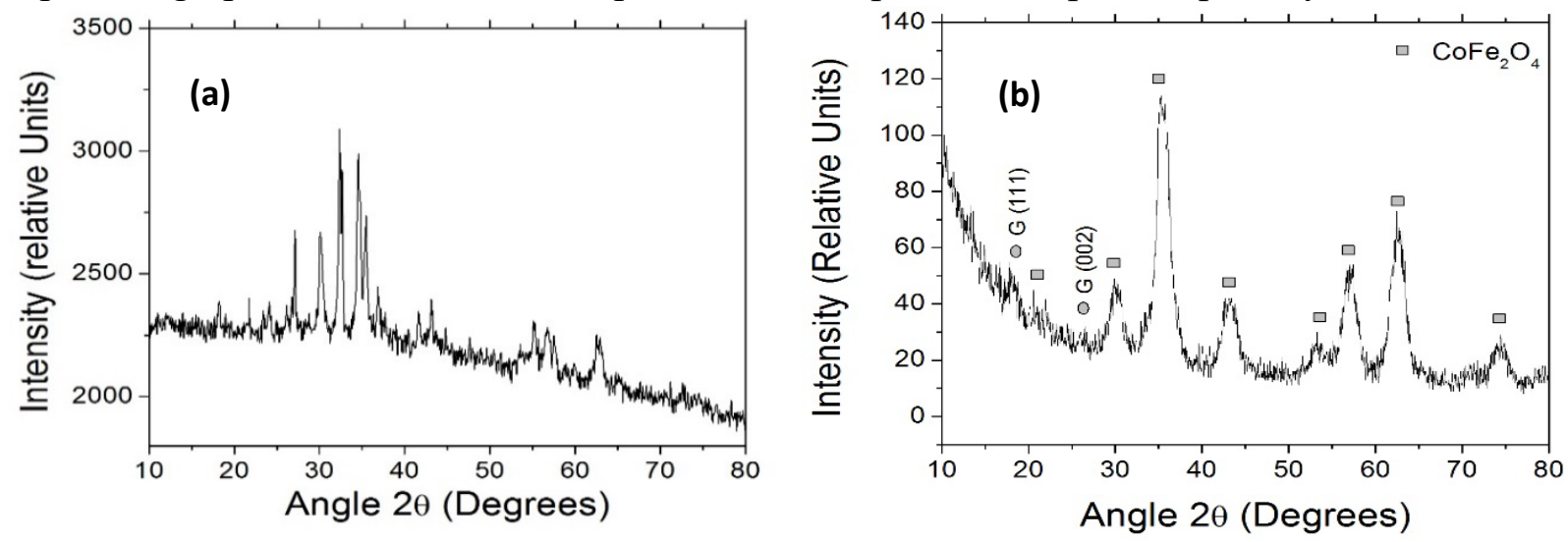

Fig. 4. XRD patterns of (a) Ba-Ferrite and (b) graphene ferrite Nano-composite

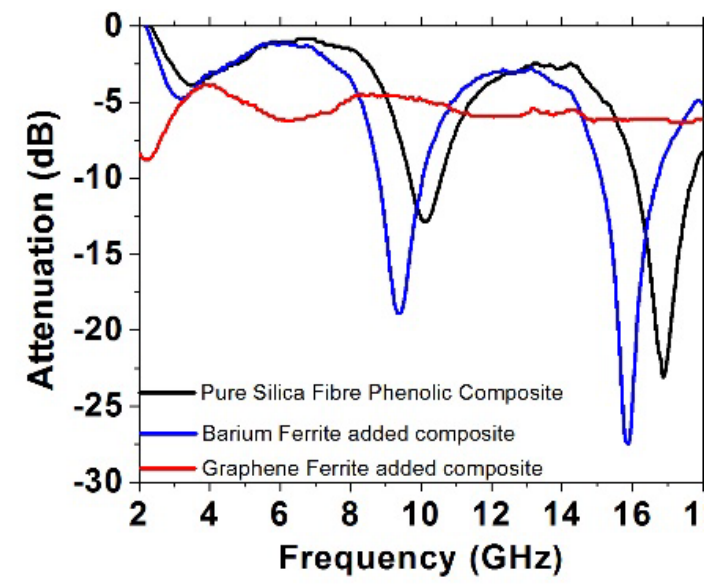

Fig. 5. RF-absorption of pure and RAM added silica fiber phenolic samples
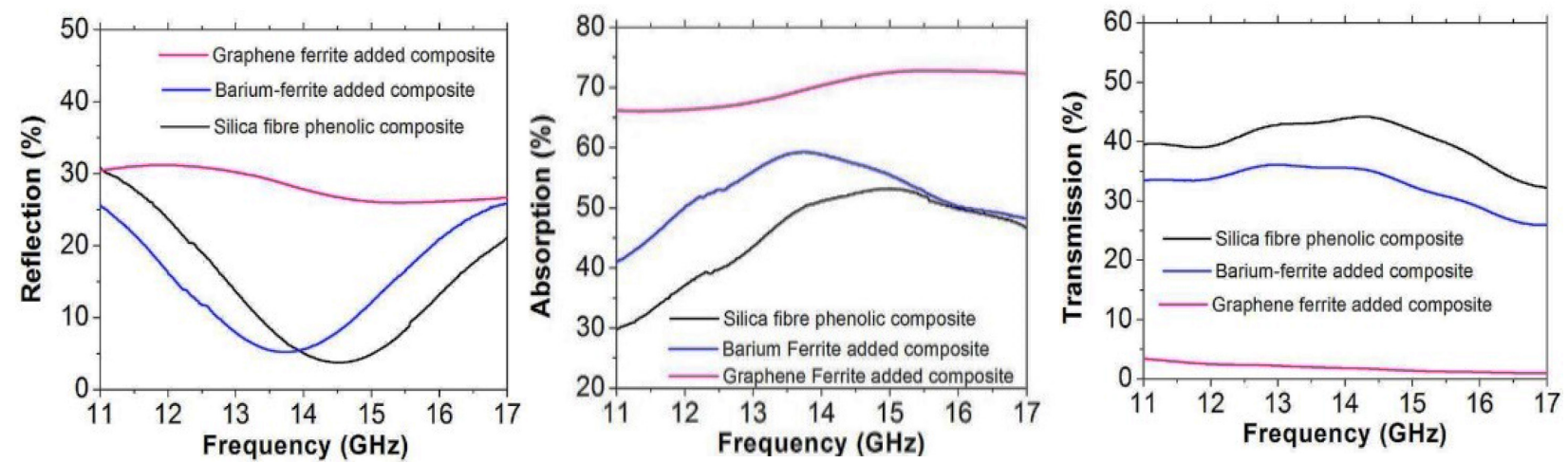

Fig. 6. Comparison of RF-absorption properties of pure and RAM added silica fiber phenolic composites

It is essential that by adding RAM the performance of the composite is not affected by variation in its physical and mechanical properties. Therefore, tensile and ablation testing was performed to ensure that efficiency of silica fiber phenolic is not compromised with the addition of RAM. Our results showed that the tensile strength of barium-ferrite added composite is higher, whereas graphene ferrite added composite is slightly lower but comparable with the pure composite. The increase in the tensile properties of barium ferrite composite and graphene ferrite nano composite is due to the addition of hard phase in the composite structure that hinders the propagation of crack. 
Despite the addition of RAM there is minute increase in density of the resultant composites especially in the case of graphene ferrite. Low thermal conductive RAMs have also proved their significance by decreasing ablation rates and back plate temperatures. Ablation testing of RAM added silica fibre phenolic composites was conducted at enthalpy $\sim 1 \mathrm{Mj} / \mathrm{Kg}$ and for exposure time of $200 \mathrm{sec}$. Barium ferrite being a low thermal conductive and a stable oxide at relatively high temperatures acted as heat resistive agent in the composite and thus gave lowest erosion rate and back plate temperature. Heat does not integrate Graphite having relatively high thermal conductivity would not have been suitable, however formation of graphene Nano-composite from graphite helped reduce the thermal conductivity which is reflected in the ablation results of graphene Nano-composite added composite.

Table 1. Tensile strength, density and ablation properties of pure and RAM added silica fiber phenolic composites

\begin{tabular}{|c|c|c|c|c|c|}
\hline & & & $\begin{array}{c}\text { Ablation } \\
\text { Rate } \\
(\mathrm{mm} / \mathrm{sec})\end{array}$ & $\begin{array}{c}\text { Back Plate } \\
\text { Temperature } \\
\left({ }^{\circ} \mathrm{C}\right)\end{array}$ \\
\hline \multicolumn{2}{|c|}{ Silica fiber phenolic } & 18.0 & 1.65 & 0.0057 & 307 \\
\hline \multirow{2}{*}{$\begin{array}{l}\text { RAM } \\
\text { Added } \\
\text { Composite }\end{array}$} & Barium Ferrite & 24.6 & 1.87 & 0.00033 & 261 \\
\hline & $\begin{array}{l}\text { Modified } \\
\text { Graphene Ferrite }\end{array}$ & 17.5 & 1.69 & 0.0011 & 269 \\
\hline
\end{tabular}

\section{Conclusions}

Two different RAMs were successfully synthesized and added in silica phenolic composites. It was established that the RF-absorption of silica phenolic composite can be enhanced with the addition of RAMs. The RF-absorption depended on the type of RAM. Graphene ferrite Nanocomposite sample which also contained ferrite nanoparticles showed broad band absorption. Further work on this type of RAM i.e. by changing the ferrite content and its amount in the silica phenolic composite can further enhance the absorption capability of the composite. Low thermal conductive RAMs also reduced ablation rates and back plate temperatures of silica phenolic composite. Different sorts of silica fibers give rise to different composite properties and the comparison of the results obtained with the literature data is deficient because these composites address sensitive zones of aerospace industry.

\section{References}

[1] K. Singh, A. Ohlan, V.H. Pham, B.R.S. Varshney, J. Jang, S. H. Hur, W.M. Choi, M. Kumar, S.K. Dhawan, B.-S. Kong and J. S. Chung, Performance of nanoarchitectured tin oxide @ reduced graphene oxide composite as a shield against electromagnetic polluting radiation,Nanoscale, 5 (2013) 2411-2420.

[2] X. Sun, J. He, G. Li, J. Tang, T. Wang, Y. Guo and H. Xue, Laminated magnetic graphene with enhanced electromagnetic wave absorption properties, J. Mater. Chem. C, 1 (2013) 765-777.

[3] P. Ohlan, K. Singh, A. Chandra and S. K. Dhawan, Microwave Absorption Behavior of Core-Shell Structured Poly (3,4-Ethylenedioxy Thiophene)-Barium Ferrite Nanocomposites, ACS App. Mat. Interf., 2 (2010) 927-933.

[4] P. Xu, X. Han, C. Wang, H. Zhao, J. Wang, X. Wang and B. Zhang, Synthesis of Electromagnetic Functionalized Nickel/Polypyrrole Core/Shell Composites, J. Phy. Chem. B, 112 (2008) 2775-2781. 
[5] L. Deng, L. Ding, K. Zhou, S. Huang, Z. Hu, B. Yang, Effects of the main active components combinations of Astragalus and Panax notoginseng on energy metabolism in brain tissues after cerebral ischemia-reperfusion in mice, J. Mag. Mag. Mat., 323 (2011) 1895-1898.

[6] W.S. Hummers, R. E. Offeman, Preparation of Graphitic Oxide, J. Am. Chem. Soc. 80 (1958) 1339-1344.

[7] Y. Zhan, F. Meng, Y. Lei, R. Zhao, J. Zhong, X. Liu, Fluorinated graphene oxide for enhanced $\mathrm{S}$ and X-band microwave absorption, Mater. Lett. 65 (2011) 1737-1740.

[8] P. Liu, Y. Huang, L. Wang, M. Zong, W. Zhang, Superb electromagnetic wave-absorbing composites based on large-scale graphene and carbon nanotube films, Mat. Lett. 107 (2013) 166169. 